\title{
Numerical Investigation on Isolated Moving Charged Droplet Evaporation in Electrostatic Field with Highly Volatile R134a
}

\author{
Jiameng Tian ${ }^{1}$, Junfeng Wang ${ }^{* 1}$, Bin Chen $^{2}$ \\ ${ }^{1}$ School of Energy and Power Engineering, Jiangsu University, Zhenjiang, PR China \\ ${ }^{2}$ State Key Laboratory of Multiphase Flow in Power Engineering, Xi'an Jiaotong University, \\ Xi'an, PR China
}

\begin{abstract}
Fundamental investigations of the charged droplet evaporation are helpful in enhancing the cooling capability and advancing the electrostatic spray model, whereas the corresponding data with the highly volatile R134a under electric field is very scarce. In this work, an improved evaporation model accounting for the effects of the corona wind, Coulombic fission and electric force under the electric field was developed and validated to evaluate the importance of the electric field on the moving charged R134a droplet evaporation for the first time. Results demonstrated that the electrostatic field can improve the charged droplet evaporation by means of the heat and mass transfer enhancement by the corona wind and counteracting the drag force from air. The droplet lifetime was shortened by $20.8 \%$ (from $105.6 \mathrm{~ms}$ to $83.6 \mathrm{~ms}$ ) when the applied voltage increased from $0 \mathrm{kV}$ to $10 \mathrm{kV}$. Exponential decay in the charged droplet lifetime when the applied voltage increased was observed.
\end{abstract}

\section{Keywords}

moving charged droplet evaporation; electrostatic field; corona wind; Coulombic fission; droplet lifetime

\section{Introduction}

Droplet heating and evaporation is a common physical phenomenon, which covers a wide range of applications. The isolated droplet rather than the droplet swarm is often chosen to investigate the evaporation mechanism, which is of vital importance to the cooling performance and the combustion efficiency. The evaporation characteristics have been widely investigated by researchers under different situations [1, 2]. In particularly, the electrostatic charged droplet evaporation has drawn the increasing attention because of its important application in electrostatic spray cooling with the superiorities of the high atomization quality, uniform droplet dispersion, precise and efficient cooling, as well as the simple system without the additional pressure fluid-supplying apparatus, which allows for the miniaturization and integration of the thermal management system for the high power electronic devices [3].

The prediction of the charged droplet evaporation characteristics subjected to an electric field was made by a non-dimensional curve considering the humidity, molality and temperature of the environmental gas [4]. Experiments on the evaporation and flame morphology of a pensile charged burning fuel droplet without the application of the external electric fields was implemented by Anderson et al. [5], in which a substantial influence of the electric charge on the vaporization rate was found. More recent work by Kourmatzis [6] extended the traditional droplet evaporation model to account for the influence of reacting sibling droplets on the vaporization and electrostatic breakup of the electrified residual droplet. The famous observation on the heat and mass transfer enhancement of fluid due to the electrostatic field was first conducted by Asakawa [7]. Afterwards, further development and explanation of this phenomenon were made by researchers, where the macroscopic 
effect of the corona wind generation [8] and the microscopic effect of the orientational alignment of fluid molecules [9] induced by an electric field were widely regarded as the primary enhancement mechanism. For the former mechanism, several enhancement factor correlations [10-12] for the Nusselt and Sherwood numbers were proposed to evaluate the heat and mass transfer enhanced by the electric field.

The objective of the present work is improving the traditional droplet evaporation model by considering the heat and mass transfer enhancement by the corona wind, droplet fragment by the Coulomb repulsion and droplet motion under the electric field. The improved model was well validated against the experimental data for the charged ethanol droplet conducted in the present work. For the first time, the charged R134a droplet evaporation with different diameters and velocities under different applied voltages in the tip-plane electrode system was simulated to evaluate the role of the electric field in the isolated moving charged droplet evaporation dynamics. Finally, the charged droplet lifetime was concluded based on the simulations to provide the first-hand data for electrostatic spray cooling with highly volatile cryogens.

\section{Methodology}

According to the Fick's law for the mass diffusion, the change rate of droplet mass during evaporation can be described by

$$
\frac{d m_{d}}{d t}=-\frac{S h}{3 S c}\left(\frac{m_{d}}{\tau_{d}}\right) B_{M}
$$

where $\tau_{D}=\rho_{d} D^{2} /\left(18 \mu_{g}\right), S h=h_{m} D / \Gamma, S c=\mu_{g} / \rho_{g} \Gamma . m_{d}, \rho_{d}$ and $D$ are the mass, density and diameter of droplet. $\rho_{g}$ and $\mu_{g}$ are the density and viscosity of the gas mixture. $\Gamma, h_{m}$ and $B_{M}$ are the binary diffusion coefficient, the mass transfer coefficient, and the Spalding mass transfer number, respectively. The lumped model with the assumption of the infinite thermal conductivity in the liquid raised by Sirignano [13], is used to simulate the heat and mass transfer between the droplet and gas. The change rate of the droplet temperature $T_{d}$ can be written as

$\frac{d T_{d}}{d t}=\frac{N u}{3 \operatorname{Pr}}\left(\frac{\theta}{\tau_{D}}\right)\left(T_{g}-T_{d}\right)+\left(\frac{L}{c_{p, d} m_{d}}\right) \frac{d m_{d}}{d t}$

where $\theta=c_{p, g} / c_{p, d}, N u=h_{c} D / \lambda_{g}, \operatorname{Pr}=\mu_{g} c_{p, g} / \lambda_{g} . T_{d}$, and $c_{p, d}$ are the temperature and specific heat of the droplet. $T_{g}, c_{p, g}$ and $\lambda_{g}$ are the temperature, specific heat and heat conductivity coefficient of the gas mixture, respectively. $h_{c}$ and $L$ are the convective heat transfer coefficient and the latent heat.

This work aims to construct the modeling of the charged droplet evaporation moving in the electric field. As shown in Figure 1, the typical tip-plane electrode system was used to produce an nonuniform electric field. The electric corona discharge often occurs when a large electric voltage is applied between the two electrodes [14].

The effects of the corona wind on the charged droplet evaporation were widely studied by researchers. Three typical enhancement factor correlations (see Table 1) defined as the ratio between the dimensionless numbers $(\mathrm{Nu}$ or $\mathrm{Sh})$ under the electric field and those $(\mathrm{Nu}$ or $S h_{0}$ ) with the absence of the electric field, were selected to evaluate the heat and mass transfer enhancement by the corona wind. $U, U_{b}, V_{c}$ and $V_{r}$ denote the applied voltage, breakdown voltage, corona wind velocity and relative velocity between gas and fluid, respectively. 


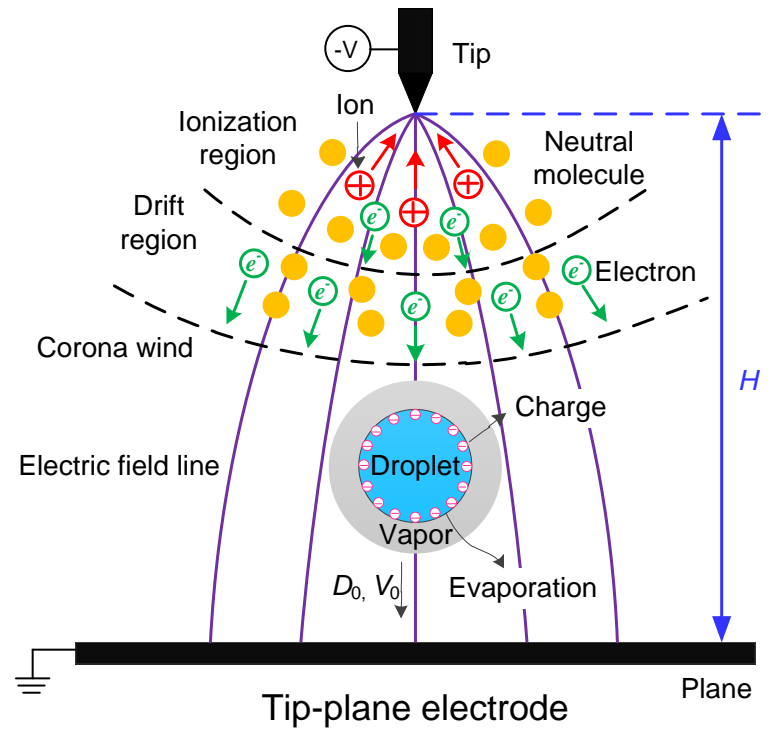

Figure 1. Schematic diagram of the charged droplet evaporation subject to the electric field with a tip-plane electrode system

Table 1 - Typical enhancement factor correlations by researchers

\begin{tabular}{cc}
\hline Authors & Correlations \\
\hline Wolny \& Kaniuk [10] & $\frac{N u}{N u_{o}}=\frac{S h}{S h_{o}}=1+0.24\left(\frac{\varepsilon_{a}}{\rho_{a}}\left(\frac{U-U_{b}}{H V_{r}}\right)^{2}\right)^{0.55}$ \\
Wangnipparnto et al. [11] & $\left.\frac{N u}{N u_{o}}=1+1.33 R e^{-0.50976}\left(\frac{U}{U_{b}}\right)^{\left(3.126 / R e^{-0.3229}\right.}\right)$ \\
Karami et al. [12] & $\frac{S h}{S h_{o}}=1+2.37 \frac{V_{c}}{V_{r}}$ \\
\hline
\end{tabular}

Droplet motion with the high initial velocity is dominated by the electric field force $\left(F_{E}\right)$ induced by the electrostatic field, the drag force $\left(F_{D}\right)$ from the surrounding gas and the gravity $\left(F_{G}\right)$, collaboratively. In consideration of the situation that the direction of the initial droplet velocity and the electric field force is consistent with that of gravity, the motion of the charged droplet can be calculated by

$m_{d} \frac{d V}{d t}=F_{E}+F_{D}+F_{G}$

where $F_{G}=m_{d} g, g=9.8 \mathrm{~m} / \mathrm{s}^{2}$ is the gravitational acceleration. The drag force $\left(F_{D}\right)$ can be computed by

$m_{d} \frac{C_{d} R e}{24 \tau_{D}}\left(V_{g}-V\right)$

where $C_{d}$ is the drag coefficient for droplet related to the Reynolds number $(R e)$ according to Morsi \& Alexander's work [15]. $V_{g}$ is the gas mixture velocity. Differing with the uniform electric field induced by parallel-plane electrode system, the highly nonuniform electric field 
can be produced with the tip-plane electrode system. Since the situation of the droplet moving along with the tip axis was considered in the present work, the electric field magnitude $(E)$ at the tip axis ignoring the space charge is a function of the axial distance $(Z)$ from tip exit to droplet for the given tip radius $\left(R_{0}\right)$ and the distance $(H)$ between two electrodes [16],

$$
\begin{aligned}
& E(Z)=\frac{H C}{Z(2 H-Z)+(H-Z) R_{0}} \\
& C=\frac{U}{\ln \left(2\left(H / R_{0}\right)^{1 / 2}\right)}
\end{aligned}
$$

The equivalent electric force $\left(F_{E}\right)$ equals to the product of the electric field magnitude $(E)$ and the charge of droplet $(Q)$ (i.e., $F_{E}=E \times Q$ ). The droplet breaks up when the droplet charge approaches the threshold (Rayleigh limit). This process related to the gas-liquid interface instability induced by Coulombic repulsion has been characterized by many researchers [1720]. Experiment photographs conducted by Duft et al. [17] shows that an initially perturbed spherical charged droplet develops conical tips at the poles. Finally, a thin filament-like jet emerges from the tips when the charge on the droplet equals to the Rayleigh limit $\left(Q_{\text {ray }}\right)$, where $Q_{\text {ray }}$ is described and formulated by Rayleigh [21] as a function of the surface tension $(\sigma)$, the droplet diameter $(D)$ and the vacuum permittivity $\left(\varepsilon_{0}\right)$,

$$
Q_{\text {ray }}=\pi\left(\varepsilon_{0} \sigma\right)^{1 / 2}(2 D)^{3 / 2}
$$

To model the fragment of the charged droplet conveniently, the complicated Coulombic fission is simplified by ignoring the detailed breakup behavior. The residual droplet has the order of $97 \%$ of the mass of the primary droplet and the order of $80 \%$ of the charge of the primary droplet [18]. In order to investigate the effect of the external applied electric filed on the charged droplet evaporation dynamics conveniently, the initial charge $\left(Q_{0}\right)$ of the primary droplet is roughly assumed to be $0.4 Q_{\text {ray }}$ according to the experiment on the charged ethanol-isooctane mixture droplet evaporation and combustion [5].

In the present simulation, the tip radius $\left(R_{0}\right)$ and distance $(H)$ between the two electrodes were set to be $0.4 \mathrm{~mm}$ and $200 \mathrm{~mm}$. Cryogen R134a with the high volatility was used to investigate the charged droplet evaporation characteristics. The initial droplet temperature was set to be $-26.1^{\circ} \mathrm{C}$, while varying initial diameters $\left(D_{0}=40,50,60 \mu \mathrm{m}\right)$ and velocities $\left(V_{0}\right.$ $=10,20,30 \mathrm{~m} / \mathrm{s}$ ) to simulate the initial droplet conditions during electrostatic R134a spray cooling. The ambient temperature and pressure were set to be the default values of $25^{\circ} \mathrm{C}$ and $0.1 \mathrm{MPa}$ except for the simulations under different ambient pressures.

Figure 2 illustrates that the charged droplet evaporation was obviously accelerated under the electric field with the applied voltage of $6 \mathrm{kV}$, compared with that without the presence of the electric field $(U=0 \mathrm{kV})$. Large discrepancies were found when using the correlations of Wangnipparnto et al. [11] and Karami et al. [12], mainly because of the absence in the Sherwood or Nusselt number correlations, as well as the difference in the electrode system. In contrast, the simulated droplet diameter using the Wolny's [10] correlation agreed well with the experimental data because of the comprehensive consideration in the heat and mass transfer enhancement under the electric field. Thus, the Wolny's correlation [10] was selected in the present simulation of R134a charged droplet. 


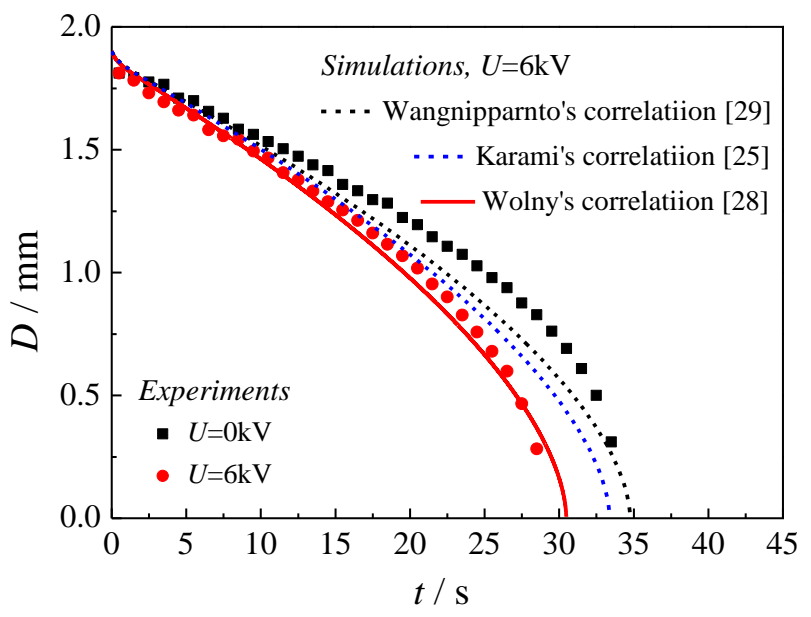

Figure 2. Comparison of the dynamic droplet diameter in the simulation and experiment

\section{Results and Discussion}

One of the key electrodynamics parameter is the applied voltage. In this contribution, the common used tip-plane electrode system was employed to investigate the droplet evaporation dynamics with different voltages $(U)$. Figure 3 displays the transient single isolated droplet velocity and diameter when exerting the applied voltages of $0,3,5,7$ and 10 $\mathrm{kV}$. Figure 3 (a) implies that the charged droplet velocities rapidly decreased first in $10 \mathrm{~ms}$, and then stabilized at their specific values under different applied voltages. Increased applied voltage produced the increased steady droplet velocity due to the large weakening effect to the drag force on droplet induced by the large electric field force. However, negligible differences in the droplet velocity were observed in the initial droplet flight period $(t$ $<10 \mathrm{~ms}$ ) with different applied voltages. Figure 3 (b) shows that more rapid decrease in the droplet diameter with the higher applied voltage was observed for the charged droplet owing to the more significant heat and mass transfer enhancement by the corona wind, as well as Coulombic fission induced by the exacerbation of the liquid-air interfacial instabilities. Besides, the higher steady droplet velocity with the larger applied voltage leaded to the enhanced convective heat transfer between the droplet and surrounding air, also yielding the increased evaporation rate. The droplet lifetime was shortened by $20.8 \%$ (from $105.6 \mathrm{~ms}$ to $83.6 \mathrm{~ms}$ ) with the implementation of the electrostatic field with the applied voltage of $10 \mathrm{kV}$.

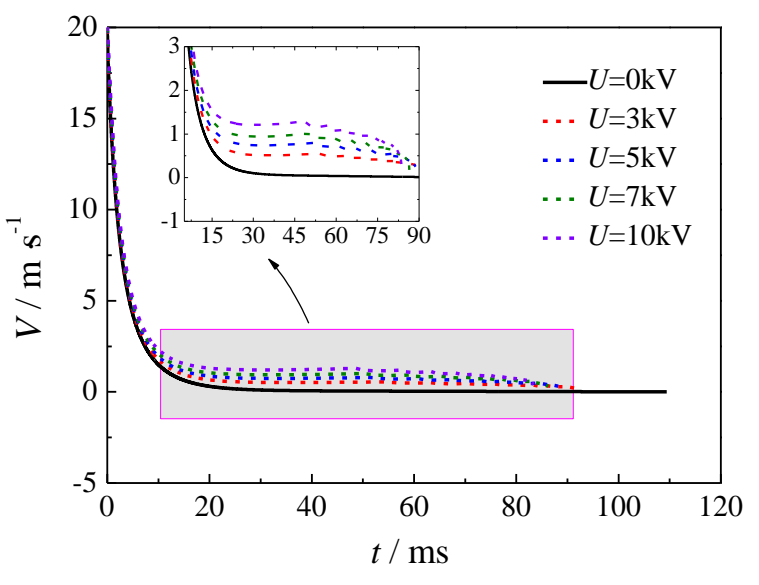

(a) droplet velocity

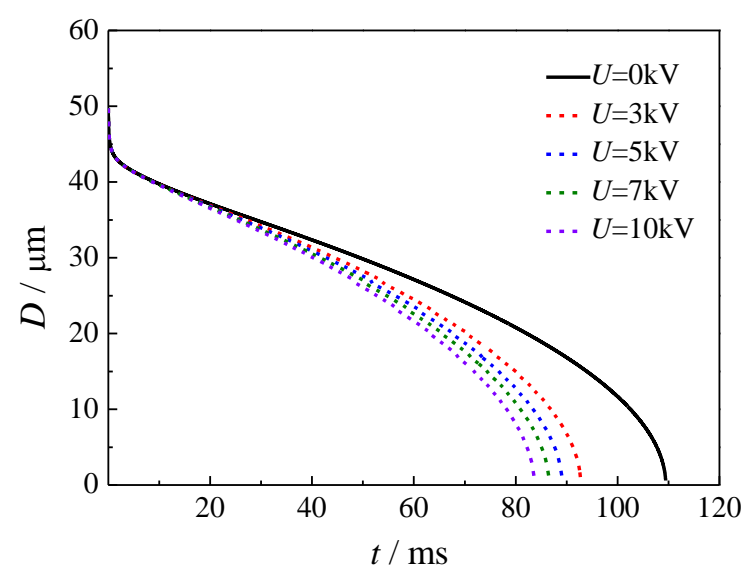

(b) droplet diameter

Figure 3. Time-varied droplet velocity and diameter with different applied voltages 
As depicted in Figure 4 (a), the droplet flight distance increased dramatically in $10 \mathrm{~ms}$, and gradually increased for the most evaporation period because of the small steady droplet velocity (Figure 3 (a)). With the increased applied voltage, the droplet velocity increased, thus yielding a large droplet flight distance. The dynamic specific charges with different applied voltages were shown in Figure 4 (b). The specific charge $(\beta)$ defined by the ratio between the charge and mass of droplet represents the droplet breakup ability induced by Coulombic repulsion on the gas-liquid interface, and affects the atomization and evaporation of electrostatic spray, as well as the droplet deposition on the heating surface, thus determining the spray cooling capability indirectly. Owing to the continue droplet evaporation, the specific charge increased as time increased. The tiny local decrease in the specific charge was produced by the sudden decreased droplet charge because of Coulombic fission.

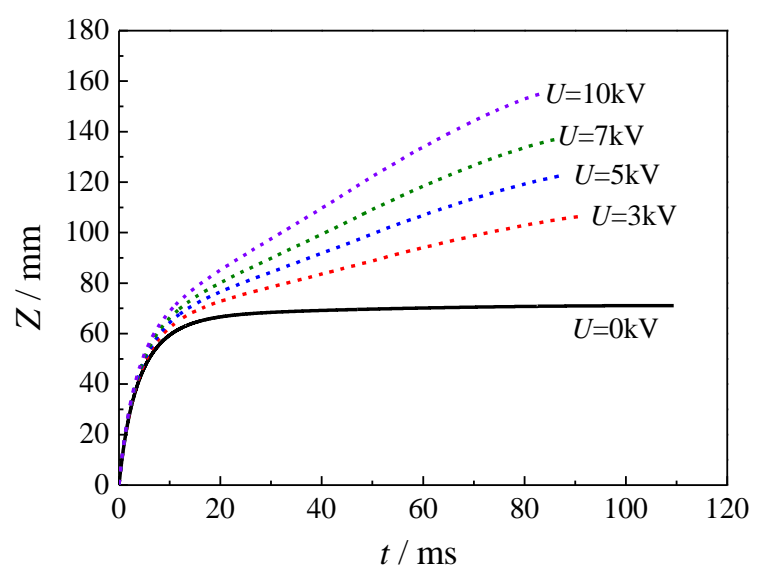

(a) droplet flight distance

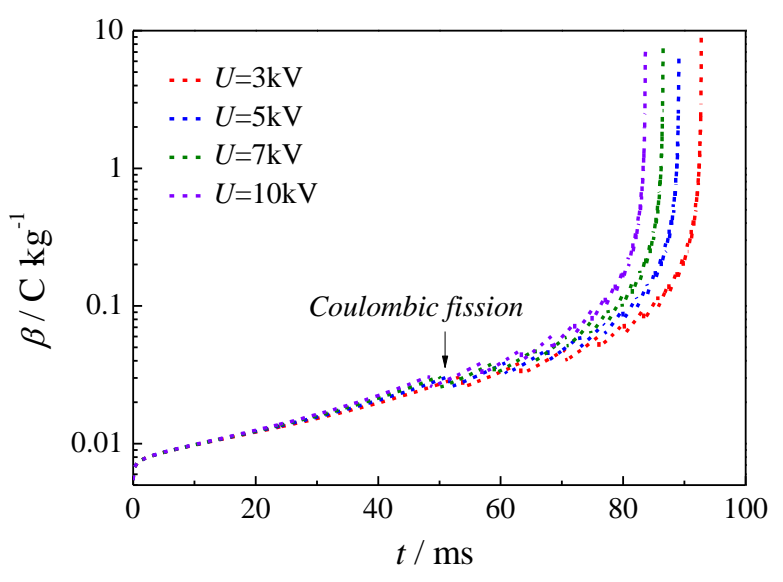

(b) droplet specific charge

Figure 4 Time-varied droplet flight distance and droplet specific charge with different applied voltages

The charged droplet evaporation is the key in electrostatic spray cooling with the highly volatile R134a because it directly determines the cooling capability. However, the fundamental data of the moving charged droplet evaporation in the presence of electric field is yet to be reported according to the author's knowledge. In this section, the droplet lifetimes in the tip-plane electrode system were concluded to provide the first-hand data for the simulation and analyze of electrostatic spray cooling.

Figure 5 (a) shows the effect of the initial droplet diameter on R134a droplet lifetime for a range of applied voltages from $0 \mathrm{kV}$ to $10 \mathrm{kV}$. Decreasing droplet lifetime when the applied voltage moves from the low to high value was found with the initial droplet diameters of 40 , 50 , and $60 \mu \mathrm{m}$ and an identical initial velocity of $20 \mathrm{~m} / \mathrm{s}$. This can be explained that the large applied voltage produces the large droplet velocity (shown in Figure 3 (a)) and enhancement factor by the corona wind [10], leading to the heat and mass transfer enhancement, thereby enhancing the evaporation. The droplet lifetime exhibited an exponential decay with the increase in the applied voltage. With an increase in the initial droplet diameter, the droplet lifetime raised because of the large droplet mass.

Figure 5 (b) depicts the effect of the initial droplet velocities $\left(V_{0}=10,20\right.$ and $\left.30 \mathrm{~m} / \mathrm{s}\right)$ on $\mathrm{R} 134 \mathrm{a}$ droplet lifetime with the same initial droplet diameter $\left(D_{0}=50 \mu \mathrm{m}\right)$. Decreasing droplet lifetimes with the three initial velocities were observed when the applied voltage increases. Interestingly, no significant effect of the initial droplet velocity on the droplet lifetime was found under the current electric field. This can be explained that the drag force 
on droplet is large enough to produce the almost identical steady droplet velocity, leading to the similar convective heat transfer and evaporation rate.

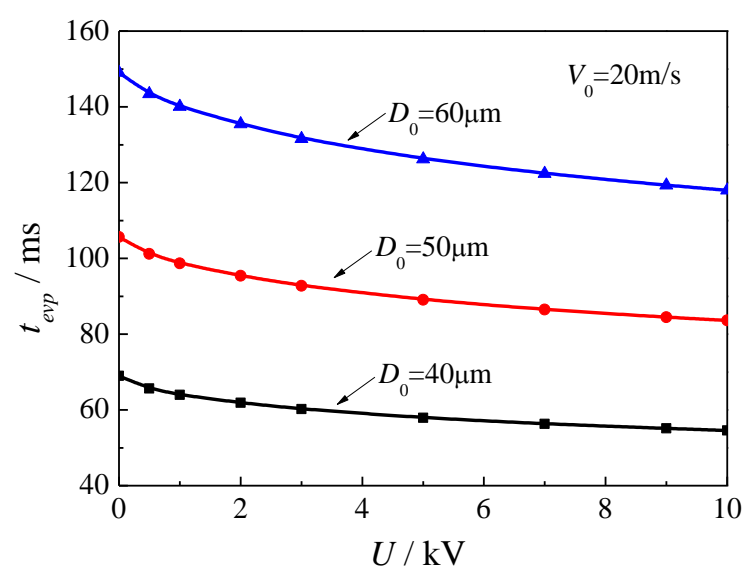

(a) effect of the initial droplet diameter

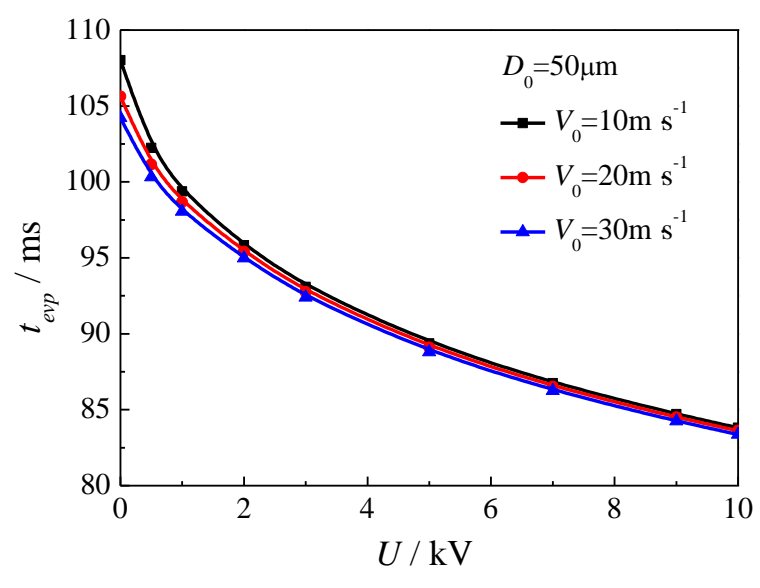

(b) effect of the initial droplet velocity

Figure 5. Effects of the initial droplet diameter and velocity on the droplet lifetime with different applied voltages

\section{Conclusions}

In this work, the traditional droplet evaporation model was improved by considering the heat and mass transfer enhancement by the corona wind, Coulombic fission and droplet motion under the electric field to investigate the isolated moving charged R134a droplet evaporation dynamics with the high volatility. The dynamic diameters of the charged ethanol droplet using the three typical enhancement factor correlations were compared, where the simulation with the Wolny's correlation shows better accuracy because of the comprehensive consideration in the heat and mass transfer enhancement under the electric field. Without the consideration of the corona wind and Coulombic fission, the charged droplet evaporation rate was underestimated.

The results of the droplet evaporation with different applied voltages demonstrated that the $\mathrm{R} 134 \mathrm{a}$ droplet diameter is very sensitive to the applied voltages. The droplet diameter decreased as the applied voltage increased because of the more significant heat and mass transfer enhancement by the corona wind, Coulombic fission induced by the exacerbation of the liquid-air interfacial instabilities, as well as the enhanced convective heat transfer induced by the high steady droplet velocity, indicating the electrostatic field can improve the charged droplet evaporation. In particular, the droplet lifetime was shortened by $20.8 \%$ (from $105.6 \mathrm{~ms}$ to $83.6 \mathrm{~ms}$ ) when the applied voltage increased from $0 \mathrm{kV}$ to $10 \mathrm{kV}$.

Decreasing droplet lifetime under the large applied voltage was found, which can be explained that the large applied voltage produces the large droplet velocity and enhancement factor by the corona wind, leading to the evaporation enhancement. An interesting phenomenon was found that the initial droplet velocity didn't change the droplet evaporation significantly, owing to the almost identical steady droplet velocity induced by the large drag force from air.

\section{Acknowledgments}

This study was supported by the Natural Science Foundation of China (No. 51976084 and No. 52036007) and the Research Foundation for Senior Talents of Jiangsu University (No. 20JDG40). 


\section{References}

[1] Xu, H., Wang, J., Tian, J., Huo, Y., Li, B., Wang, D., Zhang, W., and Yao, J., 2021, "Evaporation Characteristics and Heat Transfer Enhancement of Sessile Droplets under Non-uniform Electric Field," Exp. Therm Fluid Sci., p. 110378.

[2] Zhou, Z., Hu, M.-Y., Xin, H., Chen, B., and Wang, G.-X., 2019, "Experimental and theoretical studies on the droplet temperature behavior of R407C two-phase flashing spray," Int. J. Heat Mass Transfer, 136, pp. 664-673.

[3] Brown, N., Zhu, Y., German, G., Yong, X., and Chiarot, P., 2017, "Electrospray Deposit Structure of Nanoparticle Suspensions," J. Electrostatics, 90, pp. 67-73.

[4] Joffre, G., Prunet-Foch, B., and Cloupeau, M., 1978, "Charged droplets evaporation and motion in an electric field," Int. J. Multiphase Flow, 4(1), pp. 41-51.

[5] Anderson, E. K., Koch, J. A., and Kyritsis, D. C., 2008, "Phenomenology of electrostatically charged droplet combustion in normal gravity," Combust. Flame, 154(3), pp. 624-629.

[6] Kourmatzis, A., 2017, "Sensitivities in the Modeling of Electrostatically Charged Droplet Evaporation and Combustion," Journal of Energy Engineering, 143.

[7] ASAKAWA, 1976, "Promotion and retardation of heat transfer by electric fields," Nature, 261(5557), pp. 220-221.

[8] Park, S., Cvelbar, U., Choe, W., and Moon, S. Y., 2018, "The creation of electric wind due to the electrohydrodynamic force," Nature Communications, 9(1), p. 371.

[9] Wang, Bing-Bing, Xu, Zhi-Ming, Xiao-Dong, Yan, and Wei-Mon, 2018, "Molecular dynamics investigation on enhancement of heat transfer between electrified solid surface and liquid water," Int. J. Heat Mass Transfer.

[10] Wolny, A., and Kaniuk, R., 1996, "The Effect of Electric Field on Heat and Mass Transfer," Drying Technol., 14(2), pp. 195-216.

[11] Wangnipparnto, S., Tiansuwan, J., Kiatsiriroat, T., and Wang, C. C., 2003, "Performance analysis of thermosyphon heat exchanger under electric field," Energy Convers. Manage., 44(7), pp. 1163-1175.

[12] Karami, R., Kamkari, B., and Kashefi, K., 2011, "Investigation of Corona wind Effect on Heat and Mass Transfer Enhancement," World Academy of ence Engineering \& Technology.

[13] Sirignano, W. A., 1983, "Fuel droplet vaporization and spray combustion theory," Progress in Energy \& Combustion Science, 9(4), pp. 291-322.

[14] Wang, J., Zhu, T., Cai, Y.-X., Zhang, J.-f., and Wang, J.-b., 2020, "Review on the recent development of corona wind and its application in heat transfer enhancement," Int. J. Heat Mass Transfer, 152, p. 119545.

[15] Morsi, S. A., and Alexander, A. J., 1972, "An investigation of particle trajectories in twophase flow systems," J. Fluid Mech., 55(2), pp. 193-208.

[16] Coelho, R., and Debeau, J., 1971, "Properties of the tip-plane configuration," J. Phys. D: Appl. Phys., 4(9), pp. 1266-1280.

[17] Duft, D., Achtzehn, T., Muller, R., Huber, B. A., and Leisner, T., 2003, "Coulomb fission: Rayleigh jets from levitated microdroplets," Nature, 421(6919), pp. 128-128.

[18] Shrimpton, J. S., 2008, "Modeling dielectric charged drop break up using an energy conservation method," IEEE Transactions on Dielectrics and Electrical Insulation, 15(5), pp. 1471-1477.

[19] Hunter, H. C., and Ray, A. K., 2009, "On progeny droplets emitted during Coulombic fission of charged microdrops," Physical Chemistry Chemical Physics Pccp, 11(29), pp. 6156-6165.

[20] Li, K. Y., Tu, H., and Ray, A. K., 2005, "Charge limits on droplets during evaporation," Langmuir the Acs Journal of Surfaces \& Colloids, 21 (9), pp. 3786-3794.

[21] Rayleigh, L., 1882, "XX. On the equilibrium of liquid conducting masses charged with electricity," The London, Edinburgh, and Dublin Philosophical Magazine and Journal of Science, 14(87), pp. 184-186. 